\title{
MULTILINEAR CONVOLUTIONS DEFINED BY MEASURES ON SPHERES
}

\author{
DANIEL M. OBERLIN
}

\begin{abstract}
Let $\sigma$ be Lebesgue measure on $\Sigma_{n-1}$ and write $\sigma=\left(\sigma_{1}, \ldots, \sigma_{n}\right)$ for an element of $\Sigma_{n-1}$. For functions $f_{1}, \ldots, f_{n}$ on $\mathbf{R}$, define

$$
T\left(f_{1}, \ldots, f_{n}\right)(x)=\int_{\Sigma_{n-1}} f_{1}\left(x-\sigma_{1}\right) \cdots f_{n}\left(x-\sigma_{n}\right) d \sigma, \quad x \in \mathbf{R} .
$$

This paper partially answers the question: for which values of $p$ and $q$ is there an inequality

$$
\left\|T\left(f_{1}, \ldots, f_{n}\right)\right\|_{q} \leq C\left\|f_{1}\right\|_{p} \cdots\left\|f_{n}\right\|_{p} ?
$$
\end{abstract}

1. For a function $f$ on $\mathbf{R}$ and $t \in \mathbf{R}$, define the translate $f_{t}$ of $f$ by $f_{t}(x)=$ $f(x-t), t \in \mathbf{R}$. An $n$-linear operator $M$ taking $n$-tuples of functions on $\mathbf{R}$ to functions on $\mathbf{R}$ is called a multilinear convolution (see $[\mathbf{2}, \mathbf{3}, \mathbf{4}]$ ) if

$$
M\left(\left(f_{1}\right)_{t}, \ldots,\left(f_{n}\right)_{t}\right)=\left(M\left(f_{1}, \ldots, f_{n}\right)\right)_{t}, \quad t \in \mathbf{R} .
$$

We are interested in certain multilinear convolutions $M$ defined as follows. Let $\lambda$ be a locally finite Borel measure on $\mathbf{R}^{n}$. If $f_{1}, \ldots, f_{n}$ are continuous functions of compact support on $\mathbf{R}$, put

$$
M\left(f_{1}, \ldots, f_{n}\right)(x)=\int_{\mathbf{R}^{n}} f_{1}\left(x-x_{1}\right) \cdots f_{n}\left(x-x_{n}\right) d \lambda\left(x_{1}, \ldots, x_{n}\right), \quad x \in \mathbf{R} .
$$

Our question about such $M$ is the question of $L^{p}$-boundedness: taking $L^{p}$ norms with respect to Lebesgue measure, when do we have an inequality

$$
\left\|M\left(f_{1}, \ldots, f_{n}\right)\right\|_{q} \leq C\left\|f_{1}\right\|_{p} \cdots\left\|f_{n}\right\|_{p} ?
$$

If $\lambda$ is absolutely continuous with respect to Lebesgue measure on $\mathbf{R}^{n}$, then some results along these lines follow from [4]. This paper is concerned with the case that occurs when $\lambda$ is $\sigma$, the Lebesgue measure on the unit sphere $\Sigma_{n-1}$ in $\mathbf{R}^{n}$. Writing $\sigma=\left(\sigma_{1}, \ldots, \sigma_{n}\right)$ for an element of $\Sigma_{n-1}$, we thus define

$$
T_{n}\left(f_{1}, \ldots, f_{n}\right)(x)=\int_{\Sigma_{n-1}} f_{1}\left(x-\sigma_{1}\right) \cdots f_{n}\left(x-\sigma_{n}\right) d \boldsymbol{\sigma}, \quad x \in \mathbf{R},
$$

for, say, bounded Borel functions $f_{1}, \ldots, f_{n}$ on $\mathbf{R}$. The problem is to determine the exponent pairs $\left(\frac{1}{p}, \frac{1}{q}\right)$ such that there exists an inequality

$$
\left\|T_{n}\left(f_{1}, \ldots, f_{n}\right)\right\|_{q} \leq C(p, q)\left\|f_{1}\right\|_{p} \cdots\left\|f_{n}\right\|_{p} .
$$

Received by the editors September 21, 1987.

1980 Mathematics Subject Classification (1985 Revision). Primary 42A85, 42B15.

Partially supported by a grant from the National Science Foundation. 


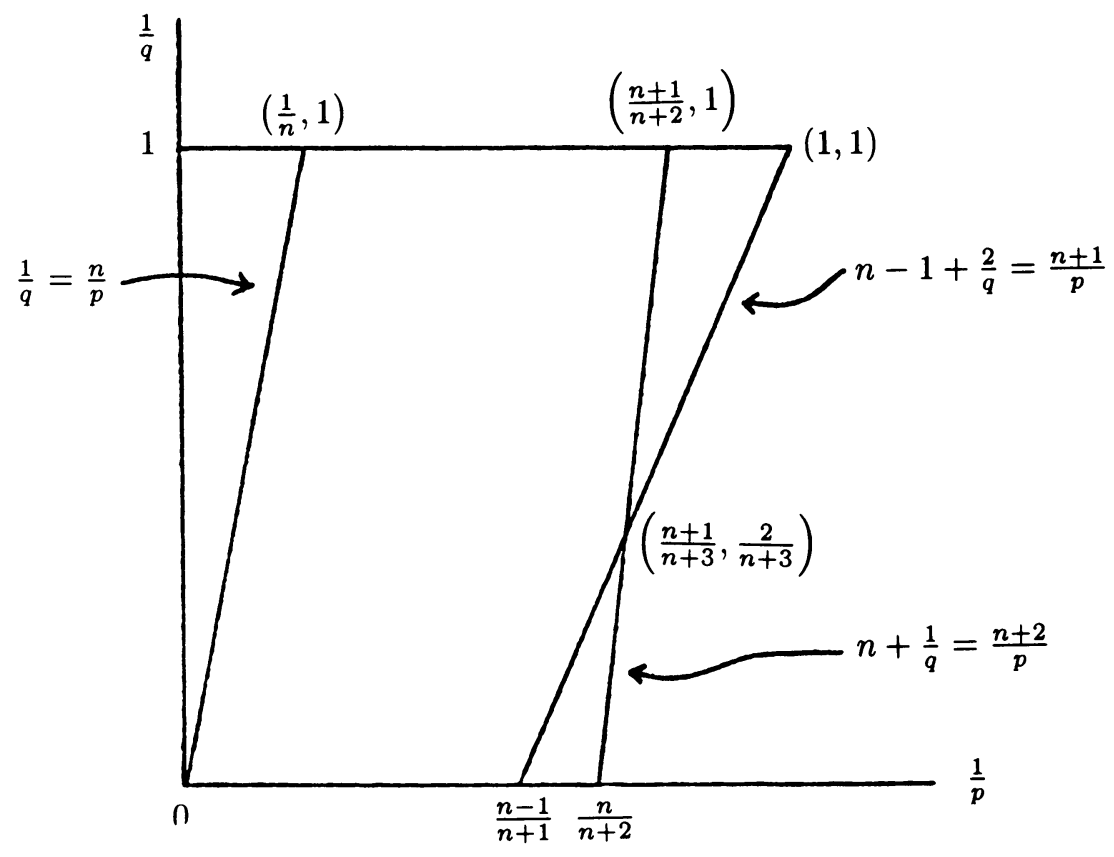

FIGURE 1

To state our result, let $R=R(n)$ be the closed convex hull in $\mathbf{R}^{2}$ of the points $(0,0),\left(\frac{1}{n}, 1\right),\left(\frac{n+1}{n+2}, 1\right),\left(\frac{n+1}{n+3}, \frac{2}{n+3}\right),\left(\frac{n-1}{n+1}, 0\right)$. (See Figure 1.)

THEOREM. If (1) holds, then $\left(\frac{1}{p}, \frac{1}{q}\right)$ lies in the region $R$. Conversely, if $\left(\frac{1}{p}, \frac{1}{q}\right)$ is in the region $R$ and not on the two closed line segments forming the right-hand boundary $D$ of $R$, then (1) holds. If the functions $f_{1}, \ldots, f_{n}$ are restricted to be characteristic functions of subsets of $\mathbf{R}$, then (1) holds also when $\left(\frac{1}{p}, \frac{1}{q}\right) \in D$.

The organization of this paper is as follows. $\S 2$ contains the proof of the necessity of the theorem's condition: that $\left(\frac{1}{p}, \frac{1}{q}\right)$ lies in $R$ if (1) holds. The rest of the paper contains the proof of the sufficiency. For technical reasons we will be concerned here with operators $S$ more general than $T_{n}$ : let $x \cdot y$ represent the scalar product of two vectors $x, y \in \mathbf{R}^{n}$, fix vectors $v_{1}, \ldots, v_{n}$ in $\mathbf{R}^{n}$, and define $S=S\left(n ; v_{1}, \ldots, v_{n}\right)$ by

$$
S\left(f_{1}, \ldots, f_{n}\right)(x)=\int_{\Sigma_{n-1}} f_{1}\left(x-v_{1} \cdot \sigma\right) \cdots f_{n}\left(x-v_{n} \cdot \sigma\right) d \boldsymbol{\sigma} .
$$

We will prove by induction on the dimension $n$ that if $v_{1}, \ldots, v_{n}$ are linearly independent, then $(\mathrm{A})$ : the inequality

$$
\left\|S\left(f_{1}, \ldots, f_{n}\right)\right\|_{q} \leq C\left\|f_{1}\right\|_{p} \cdots\left\|f_{n}\right\|_{p}
$$

holds if $\left(\frac{1}{p}, \frac{1}{q}\right)$ is in $R$ but not in $D$; and (B): the inequality

$$
\left\|S\left(I_{1}, \ldots, I_{n}\right)\right\|_{q} \leq C\left\|I_{1}\right\|_{p} \cdots\left\|I_{n}\right\|_{p}
$$

holds if $\left(\frac{1}{p}, \frac{1}{q}\right)$ is in $R$ and $I_{1}, \ldots, I_{n}$ are characteristic functions of subsets of $\mathbf{R}$. (The constants in (2) and (3) depend on $p, q$, and the choice of $v_{1}, \ldots, v_{n}$ ). 
The induction starts with the case $n=2$. This is handled in $\S 4$. We now outline the proof of (A) and (B) when $n>2$.

To prove (B) it would be sufficient, by multilinear complex interpolation using the Lorentz spaces $L^{p, 1}$ (see $[1, \S 4.4$ and 4.7]), to show that (3) holds whenever $\left(\frac{1}{p}, \frac{1}{q}\right)$ is one of the five extreme points of $R$. The point $(0,0)$ is trivial, while $\left(\frac{1}{n}, 1\right)$ is easy and is left to the reader. The point $\left(\frac{n+1}{n+2}, 1\right)$ is treated in $\S 5$, and $\left(\frac{n-1}{n+1}, 0\right)$ is considered in $\S 3$. The remaining point $\left(\frac{n+1}{n+3}, \frac{2}{n+3}\right)$ is not dealt with directly. Rather, in $\S 5$ we split the positive operator $S$ into a sum of two other positive operators: $S=S_{1}+S_{2}$. It is shown in $\S 5$ that

$$
\left\|S_{1}\left(I_{1}, \ldots, I_{n}\right)\right\|_{q} \leq C\left\|I_{1}\right\|_{p} \cdots\left\|I_{n}\right\|_{p}
$$

holds when $\left(\frac{1}{p}, \frac{1}{q}\right)=(1,1)$. Since (4) holds also whenever (3) holds, it follows that (4) holds whenever $\left(\frac{1}{p}, \frac{1}{q}\right) \in R$. It is shown in $\S 6$ that

$$
\left\|S_{2}\left(I_{1}, \ldots, I_{n}\right)\right\|_{q} \leq C\left\|I_{1}\right\|_{p} \cdots\left\|I_{n}\right\|_{p}
$$

holds when $\left(\frac{1}{p}, \frac{1}{q}\right)=\left(\frac{n}{n+2}, 0\right)$. Since (5) holds also whenever (3) holds, it follows that (5) holds whenever $\left(\frac{1}{p}, \frac{1}{q}\right) \in R$.

The proof of (A) is similar. Again, (2) is clear if $\left(\frac{1}{p}, \frac{1}{q}\right)$ is $(0,0)$ or $\left(\frac{1}{n}, 1\right)$. It is shown in $\S 5$ that (2) holds for $\frac{1}{q}=1$ if $\frac{1}{n} \leq \frac{1}{p}<\frac{n+1}{n+2}$ and in $\S 3$ that (2) holds for $\frac{1}{q}=0$ if $0 \leq \frac{1}{p}<\frac{n-1}{n+1}$. The proof is then completed by the inequalities

$$
\left\|S_{1}\left(f_{1}, \ldots, f_{n}\right)\right\|_{1} \leq C\left\|f_{1}\right\|_{1} \cdots\left\|f_{n}\right\|_{1},
$$

which is established in $\S 5$, and

$$
\left\|S_{2}\left(f_{1}, \ldots, f_{n}\right)\right\|_{\infty} \leq C\left\|f_{1}\right\|_{p} \cdots\left\|f_{n}\right\|_{p}
$$

which is established in $\S 6$ for $0 \leq \frac{1}{p}<\frac{n}{n+2}$.

2. Assume that (1) holds whenever the $f_{j}$ are characteristic functions. We will observe that the inequalities

$$
\begin{gathered}
\frac{1}{q} \leq \frac{n}{p}, \\
n-1+\frac{2}{q} \geq \frac{n+1}{p},
\end{gathered}
$$

and

$$
n+\frac{1}{q} \geq \frac{n+2}{p}
$$

must hold. These inequalities imply that $\left(\frac{1}{p}, \frac{1}{q}\right)$ lies in the region $R$. To see that (6) holds, set each $f_{j}$ equal to $I_{[-L, L]}$, the characteristic function of $[-L, L]$. Then (1) implies

$$
L^{1 / q} \leq C L^{n / p} .
$$

Letting $L \rightarrow \infty$ yields (6). Inequality (7) follows by setting $f_{j}=I_{[-\varepsilon, \varepsilon]}$ if $1 \leq j \leq$ $n-1$ and $f_{n}=I_{\left[1-2 \varepsilon^{2}, 1+2 \varepsilon^{2}\right]}$. Then (1) implies

$$
\varepsilon^{n-1+2 / q}<C \varepsilon^{(n+1) / p} .
$$


As $\varepsilon \rightarrow 0,(7)$ follows. To obtain (8), let $f_{1}$ be the characteristic function of $\left[2^{-1 / 2}-\right.$ $\left.\varepsilon^{2}, 2^{-1 / 2}+\varepsilon^{2}\right]$, let $f_{2}$ be the characteristic function of $\left[-2^{-1 / 2}-\varepsilon^{2},-2^{-1 / 2}+\varepsilon^{2}\right]$, and set $f_{j}=I_{[-\varepsilon, \varepsilon]}$ for $3 \leq j \leq n$. If $B$ is the box in $\mathbf{R}^{n}$ whose characteristic function at $\left(x_{1}, \ldots, x_{n}\right)$ is $\prod_{j=1}^{n} f_{j}\left(x_{j}\right)$, then the (surface area) measure of $(B+(t, t, \ldots, t)) \cap$ $\Sigma_{n-1}$ is of the order $\varepsilon^{n}$ if $|t| \leq \varepsilon / 2 n$. Thus (1) implies $\varepsilon^{n+1 / q} \leq C \varepsilon^{(n+2) / p}$, and this gives $(8)$.

3. Suppose that $v_{1}, \ldots, v_{n}$ are linearly independent vectors in $\mathbf{R}^{n}$. The purpose of this section is to establish the inequalities

$$
\int_{\Sigma_{n-1}} \prod_{j=1}^{n} I_{j}\left(v_{j} \cdot \sigma\right) d \sigma \leq C \prod_{j=1}^{n}\left|E_{j}\right|^{(n-1) /(n+1)}
$$

and, for $0 \leq \frac{1}{p}<\frac{n-1}{n+1}$,

$$
\int_{\Sigma_{n-1}} \prod_{j=1}^{n} f_{j}\left(v_{j} \cdot \sigma\right) d \sigma \leq C \prod_{j=1}^{n}\left\|f_{j}\right\|_{p}
$$

Here and through the paper $I_{j}$ denotes the characteristic function of $E_{j} \subseteq \mathbf{R},\left|E_{j}\right|$ denotes the Lebesgue measure of $E_{j}$, and the $f_{j}$ are nonnegative functions on $\mathbf{R}$. The constants in (9) and (10) will depend on $n$ and the choice of $v_{1}, \ldots, v_{n}$, and the constant in (10) will also depend on $p$.

We begin by observing how (10) follows from (9). Inequality (9) is equivalent to

$$
\int_{\Sigma_{n-1}} \prod_{j=1}^{n} f_{j}\left(v_{j} \cdot \sigma\right) d \sigma \leq C \prod_{j=1}^{n}\left\|f_{j}\right\|_{(n+1) /(n-1), 1}
$$

where the norms are those of the Lorentz space $L^{(n+1) /(n-1), 1}(\mathbf{R})$. If $M=$ $\max \left\{\left|v_{j} \cdot \sigma\right|: \sigma \in \Sigma_{n-1}, 1 \leq j \leq n\right\}$ and $\tilde{f}_{j}$ is the restriction of $f_{j}$ to $[-M, M]$, then the left-hand side of $(10)$ is unchanged if $f_{j}$ is replaced by $\tilde{f}_{j}$. Thus (10) is a consequence of (11) and the inequalities

$$
\left\|\tilde{f}_{j}\right\|_{(n+1) /(n-1), 1} \leq C\left\|\tilde{f}_{j}\right\|_{p} \leq C\left\|f_{j}\right\|_{p}
$$

if $\frac{n+1}{n-1}<p \leq \infty$.

We begin the proof of (9) with a lemma.

LEMMA 3.1. Suppose $E$ is a Lebesgue measurable subset of $\left\{x=\left(x_{1}, \ldots, x_{n}\right) \in\right.$ $\left.\mathbf{R}^{n}: x_{j} \geq 0,1 \leq j \leq n\right\}$. Then if $|E|$ is the Lebesgue measure of $E$, we have

$$
|E|^{n+1} \leq \frac{(n+1)^{n}}{n !} \prod_{j=1}^{n} \int_{E} x_{j} d x
$$

ProOF. It is enough to prove this in the case of a bounded $E$ with $|E|>0$. In this case, define

$$
\bar{x}_{j}=\frac{1}{|E|} \int_{E} x_{j} d x .
$$

Put

$$
l(x)=\sum_{j=1}^{n} \frac{x_{j}}{\bar{x}_{j}}, \quad x \in \mathbf{R}^{n}
$$


and

$$
T(r)=\left\{x: x_{j} \geq 0,1 \leq j \leq n ; l(x) \leq r\right\} .
$$

Define $E^{+}=E \cap T(n)$ and $E^{-}=E \sim E^{+}$. Since $\int_{E}(n-l(x)) d x=0$, we have

$$
\int_{E-}(l(x)-n) d x=\int_{E+}(n-l(x)) d x \leq \int_{T(n)}(n-l(x)) d x .
$$

Of all sets

$$
F \subseteq\left\{x: x_{j} \geq 0,1 \leq j \leq n ; l(x)>n\right\}
$$

such that

$$
\int_{F}(l(x)-n) d x \leq \int_{T(n)}(n-l(x)) d x
$$

the one with largest $|F|$ will be the set

$$
F_{0}=\left\{x: x_{j} \geq 0,1 \leq j \leq n ; n<l(x) \leq s\right\}=T(s) \sim T(n),
$$

where $s>n$ is chosen to have

$$
\int_{F_{0}}(l(x)-n) d x=\int_{T(n)}(n-l(x)) d x .
$$

Since

$$
|T(r)|=\frac{r^{n}}{n !} \prod_{j=1}^{n} \bar{x}_{j}, \quad \int_{T(r)} l(x) d x=\frac{n r^{n+1}}{(n+1) !} \prod_{j=1}^{n} \bar{x}_{j}
$$

it follows that $s=n+1$. Then

$$
|E|=\left|E^{+}\right|+\left|E^{-}\right| \leq|T(n)|+\left|F_{0}\right|=|T(s)|=\frac{(n+1)^{n}}{n !} \prod_{j=1}^{n} \bar{x}_{j} .
$$

COROLLARY 3.2. (a) If $E$ is a Lebesgue measurable subset of $\mathbf{R}^{n}$, then

$$
|E|^{n+1} \leq \frac{2^{n}(n+1)^{n}}{n !} \prod_{j=1}^{n} \int_{E}\left|x_{j}\right| d x .
$$

(b) If $w_{1}, \ldots, w_{n}$ are linearly independent in $\mathbf{R}^{n}$, there is a constant $C$ (depending on $n$ and $w_{1}, \ldots, w_{n}$ ) such that

$$
|E|^{n+1} \leq C \prod_{j=1}^{n} \int_{E}\left|w_{j} \cdot x\right| d x
$$

for measurable $E \subseteq \mathbf{R}^{n}$.

(c) If $w_{1}, \ldots, w_{n}$ are linearly independent in $\mathbf{R}^{n}$, there is a constant $C$ (depending on $n$ and $w_{1}, \ldots, w_{n}$ ) such that

$$
(\sigma(E))^{n+1} \leq C \prod_{j=1}^{n} \int_{E}\left|w_{j} \cdot \sigma\right| d \sigma
$$

for measurable $E \subseteq \Sigma_{n-1}$.

PROOF. Statement (a) is an immediate consequence of Lemma 3.1. The proof of (b) is an easy change of variables argument: With $e_{j}$ denoting the $j$ th unit vector 
in $\mathbf{R}^{n}$, define a linear mapping $T$ on $\mathbf{R}^{n}$ by $e_{j} \cdot T x=w_{j} \cdot x, 1 \leq j \leq n$. Then, writing $|T|$ for $|\operatorname{det}(T)|$,

$$
\int_{E}\left|w_{j} \cdot x\right| d x=\frac{1}{|T|} \int_{E}\left|e_{j} \cdot T x \| T\right| d x=\frac{1}{|T|} \int_{T(E)}\left|e_{j} \cdot y\right| d y .
$$

Thus

$$
\begin{aligned}
|T| \cdot|E|^{n+1} & =|T|^{-n}|T(E)|^{n+1} \leq|T|^{-n} \frac{2^{n}(n+1)^{n}}{n !} \prod_{j=1}^{n} \int_{T(E)}\left|e_{j} \cdot y\right| d y \\
& =\frac{2^{n}(n+1)^{n}}{n !} \prod_{j=1}^{n} \int_{E}\left|w_{j} \cdot x\right| d x,
\end{aligned}
$$

where the inequality follows from (a). To obtain (c), fix $E \subseteq \Sigma_{n-1}$ and define $\tilde{E}=\{r \sigma: \sigma \in E, 0 \leq r \leq 1\}$. Then

$$
|\tilde{E}|=\frac{1}{n} \boldsymbol{\sigma}(E) \quad \text { and } \quad \int_{\tilde{E}}\left|w_{j} \cdot x\right| d x=\frac{1}{n+1} \int_{E}\left|w_{j} \cdot \sigma\right| d \boldsymbol{\sigma} .
$$

Thus (c) follows from (b).

LEMMA 3.3. Fix linearly independent $v_{1}, \ldots, v_{n-1}$ in $\mathbf{R}^{n}$. Define $F: \Sigma_{n-1} \rightarrow$ $\mathbf{R}^{n-1}$ by $F(\sigma)=\left(v_{1} \cdot \sigma, \ldots, v_{n-1} \cdot \sigma\right)$ and define $D(\sigma)$ to be the determinant of the matrix whose rows are the vectors $\sigma, v_{1}, v_{2}, \ldots, v_{n-1}$. Then, with $|A|$ denoting the Lebesgue measure of a subset $A$ of $\mathbf{R}^{n-1}$, we have

$$
\int_{E}|D(\sigma)| d \sigma \leq 2|F(E)|
$$

for all $E \subseteq \Sigma_{n-1}$.

PROOF. Let $B_{n-1}$ be the closed unit ball in $\mathbf{R}^{n-1}$ and parametrize the "top half" $\Sigma_{n-1}^{+}$of $\Sigma_{n}$ by the map

$$
G: x=\left(x_{1}, \ldots, x_{n-1}\right) \mapsto\left(x_{1}, \ldots, x_{n-1}, \sqrt{1-|x|^{2}}\right)=\left(\sigma_{1}, \ldots, \sigma_{n}\right)=\sigma,
$$

$$
x \in B_{n-1} \text {. }
$$

The map $G$ carries the measure $d x / \sigma_{n}$ on $B_{n-1}$ to the measure $d \sigma$ on $\Sigma_{n-1}^{+}$. Write $v_{j}=\left(v_{j}^{1}, \ldots, v_{j}^{n}\right)$ for $1 \leq j \leq n-1$. Then the Jacobian $J_{H}$ of the map $H \doteq F \circ G$ is the determinant of the matrix $\left[v_{j}^{i}-v_{j}^{n} x_{i} / \sigma_{n}\right]_{1 \leq i, j \leq n-1}$. A little linear algebra shows that $\left|J_{H}\right|=|D(\sigma)| / \sigma_{n}$. Thus, if $y=H(x)=F(\sigma)$, then

$$
d y=\left|J_{H}\right| d x=\frac{|D(\sigma)|}{\sigma_{n}} d x=|D(\sigma)| d \sigma,
$$

at least on any subset of $\Sigma_{n-1}^{+}$where $F$ is one-to-one. A similar argument applies to $\Sigma_{n-1}^{-}$. Since $F$ is one-to-one on the two components of the complement of $\Sigma_{n-1}$ with respect to the hyperplane spanned by $v_{1}, \ldots, v_{n-1}$, the lemma follows.

ProOF OF (9). For $E \subseteq \Sigma_{n-1}$, let $v_{j} \cdot E=\left\{v_{j} \cdot \sigma: \sigma \in E\right\} \subseteq \mathbf{R}$. We will show the existence of $C$ such that

$$
\boldsymbol{\sigma}(E) \leq C \prod_{j=1}^{n}\left|v_{j} \cdot E\right|^{(n-1) /(n+1)}, \quad E \subseteq \Sigma_{n-1}
$$


Then (9) follows if $E=\left\{\sigma: v_{j} \cdot \sigma \in E_{j}, 1 \leq j \leq n\right\}$. For fixed $l$, define $F_{l}(\sigma)$ to be $\left(v_{1} \cdot \sigma, \ldots, v_{l-1} \cdot \sigma, v_{l+1} \cdot \sigma, \ldots, v_{n} \cdot \sigma\right)$. Then, using $|\cdot|$ to indicate Lebesgue measure in both $\mathbf{R}^{n-1}$ and $\mathbf{R}$,

$$
\left|F_{l}(E)\right| \leq \prod_{\substack{j=1 \\ j \neq l}}^{n}\left|v_{j} \cdot E\right|
$$

so

$$
\prod_{i=1}^{n}\left|F_{l}(E)\right| \leq \prod_{j=1}^{n}\left|v_{j} \cdot E\right|^{n-1}
$$

By Lemma 3.3,

$$
\int_{E}\left|D_{l}(\sigma)\right| d \sigma \leq 2\left|F_{l}(E)\right|,
$$

where $D_{l}(\sigma)$ is the determinant of the matrix whose rows are the vectors $\sigma, v_{1}, v_{2}, \ldots, v_{l-1}, v_{l+1}, \ldots, v_{n}$. But $D_{l}(\sigma)=w_{l} \cdot \sigma$, where $w_{l}$ is some vector orthogonal to the linear span of $\left\{v_{j}\right\}_{j \neq l}$. Since $v_{1}, \ldots, v_{n}$ are linearly independent, it follows that $w_{1}, \ldots, w_{n}$ are linearly independent also. Now (12) is a consequence of (13), (14), and (c) of Corollary 3.2.

4. Fix linearly independent $v_{1}$ and $v_{2}$ in $\mathbf{R}^{2}$ and write $e(\theta)=(\cos \theta, \sin \theta)$. Define

$$
S\left(f_{1}, f_{2}\right)(x)=\int_{0}^{2 \pi} f_{1}\left(x-v_{1} \cdot e(\theta)\right) f_{2}\left(x-v_{2} \cdot e(\theta)\right) d \theta .
$$

Consider the inequalities

$$
\begin{aligned}
& \left\|S\left(f_{1}, f_{2}\right)\right\|_{q} \leq C\left\|f_{1}\right\|_{p}\left\|f_{2}\right\|_{p} \\
& \left\|S\left(I_{1}, I_{2}\right)\right\|_{q} \leq C\left\|I_{1}\right\|_{p}\left\|I_{2}\right\|_{p} .
\end{aligned}
$$

The purpose of this section is to show that

$\left(\mathrm{A}^{\prime}\right) \quad\left(2^{\prime}\right)$ holds if $\left(\frac{1}{p}, \frac{1}{q}\right)$ is in the region $R$ but not on either of the lines $1+\frac{2}{q}=\frac{3}{p}, 2+\frac{1}{q}=\frac{4}{p}$, and

$$
\left(3^{\prime}\right) \text { holds if }\left(\frac{1}{p}, \frac{1}{q}\right) \text { is in } R .
$$

Bearing in mind the procedure for proving (A) and (B) which was laid out in $\S 1$, we begin by noting again that $\left(2^{\prime}\right)$ holds when $\left(\frac{1}{p}, \frac{1}{q}\right)=(0,0)$ (trivially) and when $\left(\frac{1}{p}, \frac{1}{q}\right)=\left(\frac{1}{2}, 1\right)$ (a consequence of Fubini's theorem and Hölder's inequality). Additionally, we recall from $\S 3$ that $\left(2^{\prime}\right)$ holds for $\frac{1}{q}=0$ when $\frac{1}{p}<\frac{1}{3}$ and that $\left(3^{\prime}\right)$ holds when $\left(\frac{1}{p}, \frac{1}{q}\right)=\left(\frac{1}{3}, 0\right)$. The next step is

LEMMA 4.1. Inequality $\left(2^{\prime}\right)$ holds when $\left(\frac{1}{p}, \frac{1}{q}\right)=\left(\frac{3}{4}, 1\right)$.

Proof. Suppose $f_{1}, f_{2} \geq 0$. Then

$$
\left\|S\left(f_{1}, f_{2}\right)\right\|_{1}=\int_{-\infty}^{\infty} f_{1}(x) \int_{0}^{2 \pi} f_{2}\left(x+v_{1} \cdot e(\theta)-v_{2} \cdot e(\theta)\right) d \theta d x
$$


Write

$$
T f(x)=\int_{0}^{2 \pi} f\left(x+v_{1} \cdot e(\theta)-v_{2} \cdot e(\theta)\right) d \theta
$$

It is enough to check that

$$
\|T f\|_{4} \leq C\|f\|_{4 / 3}
$$

Now

$$
\begin{aligned}
T f(x) & =\int_{0}^{2 \pi} f\left(x+\left|v_{1}-v_{2}\right| \cos \theta\right) d \theta \\
& =2 \int_{-\left|v_{1}-v_{2}\right|}^{\left|v_{1}-v_{2}\right|} f(x-u) \frac{d u}{\sqrt{\left|v_{1}-v_{2}\right|^{2}-u^{2}}}
\end{aligned}
$$

Since the function $h(u)=\left(\left|v_{1}-v_{2}\right|^{2}-u^{2}\right)^{-1 / 2}$ is in the Lorentz space $L^{2, \infty}$ on $\left[-\left|v_{1}-v_{2}\right|,\left|v_{1}-v_{2}\right|\right]$, inequality (15) follows from Comment 1.4 on p. 121 of [5].

Let $I$ be a subset of $[0,2 \pi]$ to be determined later and define

$$
\begin{gathered}
J=[0,2 \pi] \sim I, \quad S_{1}\left(f_{1}, f_{2}\right)(x)=\int_{I} f_{1}\left(x-v_{1} \cdot e(\theta)\right) f_{2}\left(x-v_{2} \cdot e(\theta)\right) d \theta \\
S_{2}\left(f_{1}, f_{2}\right)(x)=\int_{J} f_{1}\left(x-v_{1} \cdot e(\theta)\right) f_{2}\left(x-v_{2} \cdot e(\theta)\right) d \theta .
\end{gathered}
$$

We will show that $I$ can be chosen to have

$$
\begin{gathered}
\left\|S_{1}\left(f_{1}, f_{2}\right)\right\|_{1} \leq C\left\|f_{1}\right\|_{1}\left\|f_{2}\right\|_{1}, \\
\left\|S_{2}\left(f_{1}, f_{2}\right)\right\|_{\infty} \leq C(p)\left\|f_{1}\right\| p\left\|f_{2}\right\|_{p}, \quad \frac{1}{p}<\frac{1}{2},
\end{gathered}
$$

and

$$
\left\|S_{2}\left(I_{1}, I_{2}\right)\right\|_{\infty} \leq C\left\|I_{1}\right\|_{2}\left\|I_{2}\right\|_{2} \text {. }
$$

Since $S=S_{1}+S_{2}$ and since $S_{1}$ and $S_{2}$ are $L^{p}-L^{q}$ bounded whenever $S$ is, it will follow by interpolation from (16)-(18), from Lemma 4.1, and from the remarks preceding Lemma 4.1 that $\left(\mathrm{A}^{\prime}\right)$ and $\left(\mathrm{B}^{\prime}\right)$ hold. Since (17) follows from (18) as (10) follows from (9) in $\S 3$, we will be concerned with only (16) and (18). Write $v_{i}=r_{i} e\left(\phi_{i}\right)$ for $i=1,2$ and $v_{1}-v_{2}=r e(\phi)$, where $0 \leq \phi, \phi_{i}<2 \pi$. Note that $\phi, \phi_{1}$, and $\phi_{2}$ are all distinct since $v_{1}$ and $v_{2}$ are linearly independent. Then, if $f_{1}, f_{2} \geq 0$,

$$
\left\|S_{1}\left(f_{1}, f_{2}\right)\right\|_{1}=\int_{-\infty}^{\infty} f_{1}(x) \int_{I} f_{2}(x+r \cos (\theta-\phi)) d \theta d x .
$$

Thus (16) is true if

$$
\int_{I} f_{2}(x+r \cos (\theta-\phi)) d \theta \leq C\left\|f_{2}\right\|_{1} .
$$

The change of variables $t=\cos (\theta-\phi)$ then shows that (16) holds if $|\sin (\theta-\phi)|$ is bounded away from zero on $I$. It is therefore enough to show that (18) holds whenever $\left|\sin \left(\theta-\phi_{1}\right) \sin \left(\theta-\phi_{2}\right)\right|$ is bounded away from zero on $J$. We will show below that if $\left|\sin \left(\theta-\phi_{1}\right) \sin \left(\theta-\phi_{2}\right)\right| \geq \delta>0$ on $J$, then

$$
\left(r_{1} r_{2}\right)^{1 / 2} \delta|E| \leq 9\left|v_{1} \cdot e(E)\right|^{1 / 2}\left|v_{2} \cdot e(E)\right|^{1 / 2}, \quad E \subseteq J
$$


Here $v_{i} \cdot e(E)=\left\{v_{i} \cdot e(\theta): \theta \in E\right\}$. From this (18) will follow just as (9) followed from (12) in $\S 3$. Now $J$ can be partitioned into 9 subsets $J_{i}, 1 \leq i \leq 9$, on each of which $v_{j} \cdot e(\theta)=r_{j} \cos \left(\theta-\phi_{i}\right)$ is monotone for both $j=1$ and $j=2$. If $E_{i}=E \cap J_{i}$, then

$$
\prod_{j=1}^{2}\left|v_{j} \cdot e\left(E_{i}\right)\right|^{1 / 2}=\prod_{j=1}^{2}\left(\int_{E_{i}} r_{j}\left|\sin \left(\theta-\phi_{j}\right)\right| d \theta\right)^{1 / 2} \geq\left(r_{1} r_{2}\right)^{1 / 2} \delta\left|E_{i}\right| .
$$

This gives (19).

5. Suppose that $v_{1}, \ldots, v_{n}$ are linearly independent in $\mathbf{R}^{n}, n \geq 3$. The purpose of this section is to establish the inequalities, for $f_{1}, \ldots, f_{n} \geq 0$,

$$
\int_{-\infty}^{\infty} \int_{\Sigma_{n-1}} \prod_{j=1}^{n} I_{j}\left(x-v_{j} \cdot \sigma\right) d \sigma d x \leq C \prod_{j=1}^{n}\left|E_{j}\right|^{(n+1) /(n+2)}
$$

and, for $\frac{1}{n} \leq \frac{1}{p}<\frac{n+1}{n+2}$,

$$
\int_{-\infty}^{\infty} \int_{\Sigma_{n-1}} \prod_{j=1}^{n} f_{j}\left(x-v_{j} \cdot \sigma\right) d \sigma d x \leq C \prod_{j=1}^{n}\left\|f_{j}\right\|_{p} .
$$

We will also introduce operators $S_{1}$ and $S_{2}$ such that $S=S_{1}+S_{2}$ and show that

$$
\left\|S_{1}\left(f_{1}, \ldots, f_{n}\right)\right\|_{1} \leq C \prod_{j=1}^{n}\left\|f_{j}\right\|_{1} .
$$

As before, the constants in (20)-(22) will depend on $n$ and the choice of $v_{1}, \ldots, v_{n}$. The constant in (21) will also depend on $p$.

Recall from $\S 3$ that the linear map $T: \mathbf{R}^{n} \rightarrow \mathbf{R}^{n}$ is defined by $e_{j} \cdot T x=v_{j} \cdot x$, $x \in \mathbf{R}^{n}$. Fix a unit vector $u_{n}$ with $T u_{n}=(c, c, \ldots, c)$ for some $c \in \mathbf{R}$, and let $\left\{u_{1}, \ldots, u_{n}\right\}$ be an orthonormal basis for $\mathbf{R}^{n}$. Let $\boldsymbol{\eta}$ be Lebesgue measure on $\Sigma_{n-2}$. Parametrize (almost all of) $\Sigma_{n-1}$ by

$\sigma=\sum_{j=1}^{n-1} r \eta_{j} u_{j}+\operatorname{sgn}(r) \sqrt{1-r^{2}} u_{n} \quad$ where $\eta=\left(\eta_{1}, \ldots, \eta_{n-1}\right) \in \Sigma_{n-2},-1 \leq r \leq 1$.

Then

and, for a function $f$ on $\mathbf{R}$,

$$
d \sigma=|r|^{n-2} \frac{d r}{\sqrt{1-r^{2}}} d \eta
$$

$$
\begin{aligned}
& \int_{-\infty}^{\infty} \int_{\Sigma_{n-1}} f((x, \ldots, x)-T \sigma) d \sigma d x \\
& =\int_{-\infty}^{\infty} \int_{-1}^{1} \int_{\Sigma_{n-2}} f\left((x, \ldots, x)-\operatorname{sgn}(r) \sqrt{1-r^{2}}(c, \ldots, c)\right. \\
& \left.\quad-T\left(\sum_{k=1}^{n-1} r \eta_{k} u_{k}\right)\right) d \eta|r|^{n-2} \frac{d r}{\sqrt{1-r^{2}}} d x \\
& =\int_{-\infty}^{\infty} \int_{-1}^{1} \int_{\Sigma_{n-2}} f\left((x, \ldots, x)-r T\left(\sum_{k=1}^{n-1} \eta_{k} u_{k}\right)\right) d \eta|r|^{n-2} \frac{d r}{\sqrt{1-r^{2}}} d x
\end{aligned}
$$


In particular, the left-hand side of (21) is equal to

$$
\int_{-1}^{1} F(r)|r|^{n-2} \frac{d r}{\sqrt{1-r^{2}}}
$$

where

$$
F(r)=\int_{-\infty}^{\infty} \int_{\Sigma_{n-2}} \prod_{j=1}^{n} f_{j}\left(x-r e_{j} \cdot T\left(\sum_{k=1}^{n-1} \eta_{k} u_{k}\right)\right) d \boldsymbol{\eta} d x
$$

At the end of this section we will prove that

$$
\int_{-1}^{1} F(r)|r|^{n-2} d r \leq C \prod_{j=1}^{n}\left\|f_{j}\right\|_{1}
$$

and, if $f_{j}=I_{j}$,

$$
F(r) \leq C r^{1-n^{2} /(n+2)} \prod_{j=1}^{n}\left|E_{j}\right|^{n /(n+2)} .
$$

Let us see how (20) follows from (23) and (24): Write $\Pi$ for $\prod_{j=1}^{n}\left|E_{j}\right|$. If $\Pi \geq 1$, then, by (24),

$$
\begin{aligned}
\int_{-1}^{1} F(r)|r|^{n-2} \frac{d r}{\sqrt{1-r^{2}}} & \leq C \Pi^{n /(n+2)} \int_{-1}^{1}|r|^{(n-2) /(n+2)} \frac{d r}{\sqrt{1-r^{2}}} \\
& \leq C \Pi^{(n+1) /(n+2)} .
\end{aligned}
$$

If, on the other hand, $0<\Pi<1$, let $\delta=\Pi^{2 /(n+2)}$ and split the integral. First,

$$
\begin{aligned}
\int_{\delta-1}^{1-\delta} F(r)|r|^{n-2} \frac{d r}{\sqrt{1-r^{2}}} & \leq \delta^{-1 / 2} C \int_{\delta-1}^{1-\delta} F(r)|r|^{n-2} d r \\
& \leq \delta^{-1 / 2} C \Pi=C \Pi^{(n+1) /(n+2)}
\end{aligned}
$$

where the second inequality follows from (23). Then, from (24),

$$
\begin{aligned}
\int_{\{1-\delta \leq|r| \leq 1\}} F(r)|r|^{n-2} \frac{d r}{\sqrt{1-r^{2}}} & \leq C \Pi^{n /(n+2)} \int_{1-\delta}^{1} r^{(n-2) /(n+2)} \frac{d r}{\sqrt{1-r^{2}}} \\
& \leq C \Pi^{n /(n+2)} \delta^{1 / 2}=C \Pi^{(n+1) /(n+2)} .
\end{aligned}
$$

To deduce (21) we require the estimate

$$
F(r) \leq C r^{1-n / s} \prod_{j=1}^{n}\left\|f_{j}\right\|_{s}
$$

valid if $\frac{1}{s}=\frac{n}{n+2}-\varepsilon$ and $\varepsilon>0$ is small. This analogue of (24) will also be established at the end of this section. Fix a small $\varepsilon>0$ and put $\frac{1}{s}=\frac{n}{n+2}-\varepsilon$. Also fix $\delta$ with $0<\delta<1$. Then (25) gives

$$
\int_{-1}^{1} F(r)|r|^{n-2} \frac{d r}{\left(1-r^{2}\right)^{1-\delta}} \leq C \prod_{j=1}^{n}\left\|f_{j}\right\|_{s}
$$


We want to interpolate (26) with (23) to obtain (21) in the form

$$
\int_{-1}^{1} F(r)|r|^{n-2} \frac{d r}{\sqrt{1-r^{2}}} \leq C \prod_{j=1}^{n}\left\|f_{j}\right\|_{p}
$$

for any $\frac{1}{p}$ slightly smaller than $\frac{n+1}{n+2}$. (Since (21) holds with $\frac{1}{p}=\frac{1}{n}$, one more interpolation will then establish (21) for $\frac{1}{n} \leq \frac{1}{p}<\frac{n+1}{n+2}$.) The method of interpolating between (23) and (26) is to apply complex multilinear interpolation to the operator $\left(f_{1}, \ldots, f_{n}\right) \mapsto F(r)$ where, for the operator's range, we interpolate with change of measure between spaces $L^{1}\left([-1,1],|r|^{n-2} d r /\left(1-r^{2}\right)^{\alpha}\right), 0 \leq \alpha \leq 1-\delta$. (The theorems justifying this interpolation can be found in $\S \S 4.4$ and 5.4 of [1].) The result is that (27) holds when

We now define

$$
\frac{1}{p}=1-\frac{1}{2}\left(\frac{1}{1-\delta}\right)\left(\frac{2}{n+2}+\varepsilon\right) .
$$

$$
\begin{aligned}
S_{1}\left(f_{1}, \ldots, f_{n}\right)(x) & =\int_{\gamma-1}^{1-\gamma} \int_{\Sigma_{n-2}} \prod_{j=1}^{n} f_{j} \\
& \left(x-\operatorname{sgn}(r) c \sqrt{1-r^{2}}-e_{j} \cdot T\left(\sum_{k=1}^{n-1} r \eta_{k} u_{k}\right)\right) d \eta|r|^{n-2} \frac{d r}{\sqrt{1-r^{2}}},
\end{aligned}
$$

and $S_{2}\left(f_{1}, \ldots, f_{n}\right)(x)$ similarly, but with $\int_{\gamma-1}^{1-\gamma}$ replaced by $\int_{\{1-\gamma \leq|r| \leq 1\}}$. Here $\gamma \in(0,1)$ will be fixed subject to a further restriction in $\S 6$. Then $S=S_{1}+S_{2}$. Since

$$
\left\|S_{1}\left(f_{1}, \ldots, f_{n}\right)\right\|_{1}=\int_{\gamma-1}^{1-\gamma} F(r)|r|^{n-2} \frac{d r}{\sqrt{1-r^{2}}}
$$

if $f_{1}, \ldots, f_{n} \geq 0,(22)$ is a consequence of $(23)$.

Here is the promised proof of (23): If $f$ is a nonnegative function on $\mathbf{R}^{n}$ and $|T|$ is the absolute value of the determinant of $T$, then

$$
\begin{aligned}
|T|^{-1} & \int_{\mathbf{R}^{n}} f(x) d x \\
& =\int_{\mathbf{R}^{n}} f\left(T\left(\sum_{k=1}^{n} x_{k} u_{k}\right)\right) d x_{1} \cdots d x_{n} \\
& =\int_{-\infty}^{\infty} \int_{\mathbf{R}^{n-1}} f\left(c(x, \ldots, x)+T\left(\sum_{k=1}^{n-1} x_{k} u_{k}\right)\right) d x_{1} \cdots d x_{n-1} d x \\
& \geq|c|^{-1} \int_{-\infty}^{\infty} \int_{0}^{1} \int_{\Sigma_{n-2}} f\left((x, \ldots, x)+T\left(\sum_{k=1}^{n-1} r \eta_{k} u_{k}\right)\right) d \eta r^{n-2} d r d x
\end{aligned}
$$

Substituting $\prod_{j=1}^{n} f_{j}\left(x_{j}\right)$ for $f\left(x_{1}, \ldots, x_{n}\right)$ gives (23).

The proofs of (24) and (25) depend on the induction hypotheses: that (A) and (B) of $\S 1$ are true in dimension $n-1$. We begin by stating a fact from linear algebra which can be proved by comparing determinants:

If $v_{1}, \ldots, v_{n-1},(1,1, \ldots, 1)$ are linearly independent in $\mathbf{R}^{n}$, then the vectors $\left(\left(e_{j}-e_{1}\right) \cdot v_{1},\left(e_{j}-e_{1}\right) \cdot v_{2}, \ldots,\left(e_{j}-e_{1}\right) \cdot v_{n-1}\right), 2 \leq j \leq n$, are linearly independent in $\mathbf{R}^{n-1}$. 
In particular, the vectors $\left(\left(e_{j}-e_{1}\right) \cdot T u_{1}, \ldots,\left(e_{j}-e_{1}\right) \cdot T u_{n-1}\right), 2 \leq j \leq n$, are linearly independent in $\mathbf{R}^{n-1}$. Let $f_{j}^{r}$ denote the dilate of $f_{j}$ defined by $f_{j}^{r}(x)=f_{j}(r x)$ and define $I_{j}^{r}$ similarly. We have

$$
\begin{aligned}
F(r) & =r \int_{-\infty}^{\infty} \int_{\Sigma_{n-2}} \prod_{j=1}^{n} f_{j}^{r}\left(x-e_{j} \cdot T\left(\sum_{k=1}^{n-1} \eta_{k} u_{k}\right)\right) d \boldsymbol{\eta} d x \\
& =r \int_{-\infty}^{\infty} f_{1}^{r}(x) \int_{\Sigma_{n-2}} \prod_{j=2}^{n} f_{j}^{r}\left(x-\left(e_{j}-e_{1}\right) \cdot T\left(\sum_{k=1}^{n-1} \eta_{k} u_{k}\right)\right) d \boldsymbol{\eta} d x \\
& =r \int_{-\infty}^{\infty} f_{1}^{r}(x) \int_{\Sigma_{n-2}} \prod_{j=2}^{n} f_{j}^{r}\left(x-w_{j} \cdot \eta\right) d \boldsymbol{\eta} d x
\end{aligned}
$$

where $w_{j}=\left(\left(e_{j}-e_{1}\right) \cdot T u_{1}, \ldots,\left(e_{j}-e_{1}\right) \cdot T u_{n-1}\right), 2 \leq j \leq n$. Define

$$
S_{n-1}\left(f_{2}, \ldots, f_{n}\right)(x)=\int_{\Sigma_{n-2}} \prod_{j=2}^{n} f_{j}\left(x-w_{j} \cdot \eta\right) d \boldsymbol{\eta} .
$$

Since $\left\|I_{j}^{r}\right\|_{p}=|r|^{-1 / p}\left\|I_{j}\right\|_{p},(24)$ follows from

$$
\left\|S_{n-1}\left(I_{2}^{r}, \ldots, I_{n}^{r}\right)\right\|_{(n+2) / 2} \leq C \prod_{j=2}^{n}\left\|I_{j}^{r}\right\|_{(n+2) / n},
$$

which is the case $\left(\frac{1}{p}, \frac{1}{q}\right)=\left(\frac{n}{n+2}, \frac{2}{n+2}\right)$ of (3) in dimension $n-1$. Finally, (25) follows similarly from

$$
\left\|S_{n-1}\left(f_{2}^{r}, \ldots, f_{n}^{r}\right)\right\|_{s^{\prime}} \leq C \prod_{j=2}^{n}\left\|f_{j}^{r}\right\|_{s}, \quad \frac{1}{s}+\frac{1}{s^{\prime}}=1,
$$

(that is the case $\left(\frac{1}{p}, \frac{1}{q}\right)=\left(\frac{1}{s}, \frac{1}{s^{\prime}}\right)$ of (2) in dimension $\left.n-1\right)$ which is true because $\left(\frac{1}{s}, \frac{1}{s^{\prime}}\right) \in R(n-1)$ if $\frac{1}{s}=\frac{n}{n+2}-\varepsilon$ and $\varepsilon>0$ is small.

6. With $S_{2}$ as defined in $\S 5$, the purpose of this section is to prove the inequalities

$$
\left\|S_{2}\left(I_{1}, \ldots, I_{n}\right)\right\|_{\infty} \leq C \prod_{j=1}^{n}\left|E_{j}\right|^{n /(n+2)},
$$

and, if $0 \leq \frac{1}{p}<\frac{n}{n+2}$,

$$
\left\|S_{2}\left(f_{1}, \ldots, f_{n}\right)\right\|_{\infty} \leq C \prod_{j=1}^{n}\left\|f_{j}\right\|_{p} .
$$

As before, the constants will depend on $n, v_{1}, \ldots, v_{n}$, and (in (29)) on $p$. Now (29) follows from (28) as (10) followed from (9) in $\S 3$. For $0<\delta<1$, let $M(\delta)=\{\sigma \in$ $\left.\Sigma_{n-1}:\left|\sigma \cdot u_{n}\right| \leq \delta\right\}$. Since

$$
S_{2}\left(I_{1}, \ldots, I_{n}\right)(x)=\int_{M\left(\sqrt{2 \gamma-\gamma^{2}}\right)} \prod_{j=1}^{n} I_{j}\left(x-v_{j} \cdot \sigma\right) d \sigma
$$


(28) will follow when we show that for some fixed $\delta=\delta\left(v_{1}, \ldots, v_{n}\right) \in(0,1)$ we have

$$
\int_{M(\delta)} \prod_{j=1}^{n} I_{j}\left(v_{j} \cdot \sigma\right) d \sigma \leq C \prod_{j=1}^{n}\left|E_{j}\right|^{n /(n+2)} .
$$

For $j=1, \ldots, n$ define $\tilde{v}_{j}=v_{j} /\left|v_{j}\right|$. For $\delta>0$ define $N(\delta)$ to be the set

$$
\left\{\sigma \in \Sigma_{n-1}:\left|\sigma-\tilde{v}_{j}\right|,\left|\sigma+\tilde{v}_{j}\right|>\delta \text { for } 1 \leq j \leq n\right\} .
$$

Since $v_{j} \cdot u_{n}=e_{j} \cdot T u_{n}=c \neq 0$ for $1 \leq j \leq n$, there is some positive $\delta$ such that $M(\delta) \subseteq N(\delta)$. We will show, then, that

$$
\int_{N(\delta)} \prod_{j=1}^{n} I_{j}\left(v_{j} \cdot \sigma\right) d \sigma \leq C \prod_{j=1}^{n}\left|E_{j}\right|^{n /(n+2)} .
$$

Let $S_{j}$ be the linear span of $\left\{v_{1}, \ldots, v_{j-1}, v_{j+1}, \ldots, v_{n}\right\}$. Since $\bigcap_{j \neq j_{0}} S_{j}$ is the set of scalar multiples of $v_{j_{0}}$, there is an $\varepsilon>0$ such that, for any $j_{0}$,

$\operatorname{dist}\left(\sigma, S_{j}\right)<\varepsilon$ for all $j \neq j_{0}$ implies $\min \left\{\left|\sigma+\tilde{v}_{j_{0}}\right|,\left|\sigma-\tilde{v}_{j_{0}}\right|\right\}<\delta \quad\left(\sigma \in \Sigma_{n-1}\right)$.

In particular,

$$
N(\delta) \subseteq \bigcup_{j=1}^{n}\left\{\sigma \in \Sigma_{n-1}: \operatorname{dist}\left(\sigma, S_{j}\right) \geq \varepsilon\right\}
$$

Thus, for fixed $E_{1}, \ldots, E_{n}$, there is some $j_{0}\left(1 \leq j_{0} \leq n\right)$ such that if

$$
N\left(\delta, j_{0}\right)=N(\delta) \cap\left\{\sigma: \operatorname{dist}\left(\sigma, S_{j_{0}}\right) \geq \varepsilon\right\}
$$

then

$$
\int_{N(\delta)} \prod_{j=1}^{n} I_{j}\left(v_{j} \cdot \sigma\right) d \sigma \leq n \int_{N\left(\delta, j_{0}\right)} \prod_{j=1}^{n} I_{j}\left(v_{j} \cdot \sigma\right) d \sigma .
$$

So fix $j_{0}$. Inequality $(30)$ will follow from

$$
\int_{N\left(\delta, j_{0}\right)} \prod_{j=1}^{n} I_{j}\left(v_{j} \cdot \sigma\right) d \sigma \leq C \prod_{j=1}^{n}\left|E_{j}\right|^{n /(n+2)} .
$$

Let $\left\{w_{1}, \ldots, w_{n-1}, \tilde{v}_{j_{0}}\right\}$ be an orthonormal basis for $\mathbf{R}^{n}$. Parametrize (almost all of) $\Sigma_{n-1}$ by

$\sigma=\sum_{k=1}^{n-1} r \eta_{k} w_{k}+\operatorname{sgn}(r) \sqrt{1-r^{2}} \tilde{v}_{j_{0}} \quad$ where $\eta=\left(\eta_{1}, \ldots, \eta_{n-1}\right) \in \Sigma_{n-2},-1 \leq r \leq 1$.

Then the inequalities $\left|\sigma \pm \tilde{v}_{j_{0}}\right|>\delta$ imply $|r|>\alpha$ for some $\alpha=\alpha(\varepsilon)>0$. Thus, if $V=\left\{\sigma: \operatorname{dist}\left(\sigma, S_{j_{0}}\right) \geq \varepsilon\right\}$, then

$$
\begin{aligned}
\int_{N\left(\delta, j_{0}\right)} & \prod_{j=1}^{n} I_{j}\left(v_{j} \cdot \sigma\right) d \sigma \\
\leq & \int_{\{\alpha \leq|r| \leq 1\}} \int_{\Sigma_{n-2}} \prod_{j \neq j_{0}} I_{j}\left(v_{j} \cdot\left[\sum_{k=1}^{n-1} r \eta_{k} w_{k}+\operatorname{sgn}(r) \sqrt{1-r^{2}} \tilde{v}_{j_{0}}\right]\right) I_{V}(\eta, r) d \eta \\
& \cdot I_{j_{0}}\left(\left|v_{j o}\right| \operatorname{sgn}(r) \sqrt{1-r^{2}}\right)|r|^{n-2} \frac{d r}{\sqrt{1-r^{2}}} .
\end{aligned}
$$


Then (31) will follow if the right-hand side of the inequality above can be bounded by

$$
C \prod_{j=1}^{n}\left|E_{j}\right|^{n /(n+2)}
$$

To simplify notation we work only with $\int_{\alpha}^{1}$ the integral $\int_{-1}^{-\alpha}$ is treated similarly. Write

$$
F(r)=\int_{\Sigma_{n-2}} \prod_{j \neq j_{0}} I_{j}\left(v_{j} \cdot\left[\sum_{k=1}^{n-1} r \eta_{k} w_{k}+\sqrt{1-r^{2}} \tilde{v}_{j_{0}}\right]\right) I_{V}(\eta, r) d \eta
$$

Then the desired inequality is

$$
\int_{\alpha}^{1} F(r) I_{j_{0}}\left(\left|v_{j_{0}}\right| \sqrt{1-r^{2}}\right) r^{n-2} \frac{d r}{\sqrt{1-r^{2}}} \leq C \prod_{j=1}^{n}\left|E_{j}\right|^{n /(n+2)} .
$$

Since

$$
\int_{\alpha}^{1} I_{j_{0}}\left(\left|v_{j_{0}}\right| \sqrt{1-r^{2}}\right) r^{n-2} \frac{d r}{\sqrt{1-r^{2}}} \leq C\left|E_{j_{0}}\right|
$$

the duality between the Lorentz spaces $L^{(n+2) / n, 1}$ and $L^{(n+2) / 2, \infty}$ (on the interval $[\alpha, 1]$ with the measure $\left.r^{n-2} d r / \sqrt{1-r^{2}}\right)$ shows that it is enough to prove the following for $\lambda>0$ :

(32) if $E=\{r \in[\alpha, 1]: F(r)>\lambda\}$, then $\lambda\left(\int_{\alpha}^{1} I_{E}(r) r^{n-2} \frac{d r}{\sqrt{1-r^{2}}}\right)^{2 /(n+2)}$

$$
\leq C \prod_{j \neq j_{0}}\left|E_{j}\right|^{n /(n+2)} .
$$

To prove (32), let us assume for the moment the inequalities

$$
\begin{gathered}
F(r) \leq C \prod_{j \neq j_{0}}\left|E_{j}\right|^{(n-2) / n}, \quad \alpha \leq r \leq 1, \\
\int_{\alpha}^{1} F(r) r^{n-2} \frac{d r}{\sqrt{1-r^{2}}} \leq C \prod_{j \neq j_{0}}\left|E_{j}\right| .
\end{gathered}
$$

Write $I$ for the integral

If $I \neq 0$, then

$$
\int_{\alpha}^{1} I_{E}(r) r^{n-2} \frac{d r}{\sqrt{1-r^{2}}}
$$

$$
\lambda \leq C \prod_{j \neq j_{0}}\left|E_{j}\right|^{(n-2) / n}
$$

follows from (33). Also, the inequality

$$
\lambda I \leq \int_{\alpha}^{1} F(r) r^{n-2} \frac{d r}{\sqrt{1-r^{2}}} \leq C \prod_{j \neq j_{0}}\left|E_{j}\right|
$$

follows from (34). These last two inequalities then yield (32) in the form

$$
\lambda^{1+2 / n} I^{2 / n} \leq C \prod_{j \neq j_{0}}\left|E_{j}\right|
$$


To prove (33) we begin by defining vectors $u_{j} \in \mathbf{R}^{n-1}, j \neq j_{0}$, by $u_{j}=\left(v_{j}\right.$. $\left.w_{1}, \ldots, v_{j} \cdot w_{n-1}\right)$. We will need to know that these $n-1$ vectors are linearly independent in $\mathbf{R}^{n-1}$. This is true because the absolute value of the determinant of the matrix whose rows are the vectors $u_{j}$ is the same as the absolute value of the determinant of the matrix $\tilde{V} \cdot \tilde{W}$. Here $\tilde{V}$ is the (nonsingular) matrix whose rows are the vectors $v_{1}, \ldots, v_{j_{0}-1}, v_{j_{0}+1}, \ldots, v_{n}, \tilde{v}_{j_{0}}$ and $\tilde{W}$ is the matrix whose columns are the (orthonormal) vectors $w_{1}, \ldots, w_{n-1}, \tilde{v}_{j_{0}}$.

Now for $j \neq j_{0}$

$$
v_{j} \cdot\left[\sum_{k=1}^{n-1} r \eta_{k} w_{k}+\sqrt{1-r^{2}} \tilde{v}_{j_{0}}\right]=r u_{j} \cdot \eta+\sqrt{1-r^{2}} v_{j} \cdot \tilde{v}_{j_{0}}
$$

so

$$
F(r) \leq \int_{\Sigma_{n-2}} \prod_{j \neq j_{0}} I_{j}\left(r u_{j} \cdot \eta+\sqrt{1-r^{2}} v_{j} \cdot \tilde{v}_{j_{0}}\right) d \boldsymbol{\eta} .
$$

By (9) in dimension $n-1$, the right-hand side of the inequality above is bounded by

$$
C r^{-(n-1)(n-2) / n} \prod_{j \neq j_{0}}\left|E_{j}\right|^{(n-2) / 2} .
$$

Since $\alpha \leq r \leq 1$, this yields (33). To obtain (34), we begin by noting that

$$
\int_{\alpha}^{1} F(r) r^{n-2} \frac{d r}{\sqrt{1-r^{2}}} \leq \int_{V} \prod_{j \neq j_{0}} I_{j}\left(v_{j} \cdot \sigma\right) d \sigma
$$

Let $D(\sigma)$ be the determinant of the matrix whose rows are the vector $\sigma$ and the vectors $v_{j}, j \neq j_{0}$. It follows from Lemma 3.3 that

$$
\int_{V} \prod_{j \neq j_{0}} I_{j}\left(v_{j} \cdot \sigma\right)|D(\sigma)| d \sigma \leq 2 \prod_{j \neq j_{0}}\left|E_{j}\right|
$$

Since

$$
V=\left\{\sigma: \operatorname{dist}\left(\sigma, S_{j_{0}}\right) \geq \varepsilon\right\}
$$

and since $D(\sigma)=0$ if and only if $\sigma \in S_{j_{0}}$, it follows that

$$
|D \sigma| \geq \beta=\beta(\varepsilon)>0 \quad \text { if } \sigma \in V .
$$

Together with (35) and (36), this gives (34).

\section{REFERENCES}

1. J. Bergh and J. Löfström, Interpolation spaces, Springer-Verlag, Berlin, 1976.

2. R. R. Coifman and Y. Meyer, Fourier analysis of multilinear convolutions, ('aldéron's theorem, and analysis on Lipschitz curves, Euclidean Harmonic Analysis (College Park, Md., 1979), Lecture Notes in Math., vol. 779, Springer-Verlag, Berlin, 1980, pp. 104- 122.

3. M. Murray, Multilinear convolutions and transference, Michigan Math. J. 31 (1984), 321-330.

4. D. Oberlin, A multilinear Young's inequality, Canad. Math. Bull. (to appear).

5. E. Stein, Singular integrals and differentiability properties of functions, Princeton Univ. Press, Princeton, N.J., 1970.

Department of Mathematics, Florida State University, Tallahassee, FLORIDA 32306 